\title{
EL CONSUMO EN LOS HOGARES ESPAÑOLES DESDE LA PERSPECTIVA LOCAL
}

\author{
339:311
}

por

Ignacio Ballester Ros

SUMARIO: I. INTRODUCCION.-II. LA VALORACION DEL CONSUMO POR HOGAR Y POR PERSONA: 1. EN LAS REGIONES HISTÓRICas. 2. EN las Provincias. 3. EN los Municipios, Según sus Niveles de POBLACION.-III. LA EVOLUCION DEL CONSUMO POR PERSONA EN LOS CONJUNTOS URBANO Y SUBURBANO.

\section{INTRODUCCION}

El Instituto Nacional de Estadística acaba de publicar una monografía en la que describe la metodología utilizada y presenta los resultados de la «Encuesta de presupuestos familiares», realizada, a lo largo del año, desde el mes de julio de 1973 al mes de junio de 1974, tomando como base las informaciones facilitadas por más de 24.000 hogares, que comprenden más de 95.000 personas.

El mencionado Instituto ha llevado a cabo varias encuestas de esta naturaleza en 1958, 1964 y 1968; pero la última, a la que nos referimos en esta crónica, es la más importante de las realizadas por su amplitud, ya que no se ha limitado sólo a analizar el consumo de los hogares, sino que ha abordado también la determinación de los ingresos y del ahorro, en función del número de perso- 
nas que componen el hogar, del número de miembros que perciben ingresos y del nivel académico y categoría socioeconómica del sustentador principal.

Casi 180 páginas de la publicación se dedican a la exposición del enfoque, características y objetivos de la encuesta y de la metodología aplicada, así como a definir las variables y demás características estudiadas. Igualmente se trata con toda amplitud el diseño de la muestra utilizada, el programa de trabajos de campo, el tratamiento de los datos, ya que, por vez primera, se han utilizado ordenadores electrónicos para elaborar los resultados de la encuesta. También se formulan consideraciones sobre los resultados obtenidos y, finalmente, se comparan estos resultados con los deducidos de las encuestas anteriores.

Como en la propia monografía se resalta, a las encuestas de presupuestos familiares se les asigna «un importante papel en el marco de las estadísticas básicas al servicio de la economía».

En primer lugar permiten conocer la estructura y evolución del consumo de las economías domésticas, que aconstituye la casi totalidad del consumo privado», y atener una amplia idea de la renta nacional, a través del gasto de los consumidores, que resulta de gran utilidad por cuanto permite contrastar las cifras de renta con los ingresos y la producción..

En segundo término, estas encuestas, «al proporcionar información sobre cantidades físicas consumidas, facilitan la determinación de la demanda» y permiten aestablecer previsiones de consumo futuros y aplanificar la producción y el comercio exterior a largo plazo».

En tercer lugar son esenciales para la elaboración del índice del coste de la vida, al proporcionar las ponderaciones y los precios del año base, $y$ aconstituyen un índice de precios, elemento deflactor que hace posible detectar los desajustes entre la oferta y la demanda y son muy eficaces para una política de control de precios».

Son útiles, a su vez, para la formación de la Contabilidad nacional, que necesita de la mayor información posible sobre el consumo de las economías domésticas; pero es, además, necesario conocer, más allá de los objetivos puramente económicos, el comportamiento social de los hogares, que no son sólo elementos de consumo. 
Los objetivos inmediatos de esta encuesta son: a) Proporcionar la información necesaria para formar un nuevo sistema de índices del coste de la vida, actualizando la acesta de la compra»; b) Conocer la estructura general del presupuesto de los hogares, sus ingresos, su empleo en los diferentes bienes y servicios de consumo y el ahorro; c) Facilitar la estimación global del consumo de bienes y servicios en las cuentas nacionales, y $d$ ) Permitir el análisis sociológico y el estudio de las homogeneidades y contrastes entre los diferentes estratos socioeconómicos.

Dada la abundante información contenida en la monografía, se limita esta crónica a la sola exposición y comentario de las cifras expresivas del consumo, atendiendo especialmente a su valoración y distribución en las Regiones históricas, Provincias y Municipios y a su evolución, deducida de los resultados de las distintas encuestas realizadas. •

Queda un acervo informativo muy amplio para que los estudiosos profundicen en su análisis desde otros puntos de vista, económicos y sociológicos, en función de las características contempladas en la encuesta, especialmente por lo que se refiere a la distribución del consumo por grupos de gastos, que no podemos abordar por limitaciones de espacio.

\section{LA VALORACION DEL CONSUMO POR HOGAR Y POR PERSONA}

\section{EN LAS RegIONES históricas}

Se ha definido al hogar como ala persona o conjunto de personas que ocupan en común una vivienda familar o parte de ella y consumen alimentos y otros bienes con cargo a un mismo presupuesto».

Se han fijado, en primer término, como resultados primarios de la encuesta: el gasto anual medio de consumo por hogar para el conjunto nacional y con referencia al período de la encuesta en 267.255 pesetas, $y$ el gasto anual medio de consumo por persona en 71.715 pesetas.

Para cada una de las Regiones históricas, el gasto anual medio de consumo alcanza las cuantías que se expresan a continuación. Igualmente se han obtenido los números índices correspondientes. 


\begin{tabular}{|c|c|c|c|c|}
\hline & \multicolumn{2}{|c|}{$\begin{array}{l}\text { CIFRAS ABSOLUTAS } \\
\text { (PESETAS) }\end{array}$} & \multicolumn{2}{|c|}{$\begin{array}{l}\text { NUMEROS INDICES. BASE: } \\
\text { CONSUMO NACIONAL }=100\end{array}$} \\
\hline & Por hogar & Por persona & Por hogar & Por persona \\
\hline 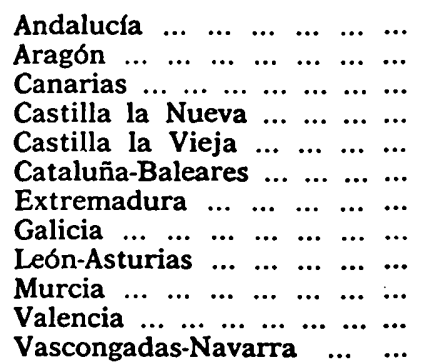 & $\begin{array}{l}224.483 \\
255.314 \\
299.630 \\
317.035 \\
247.823 \\
305.739 \\
177.279 \\
224.384 \\
224.384 \\
218.445 \\
248.432 \\
335.358\end{array}$ & $\begin{array}{l}55.899 \\
75.646 \\
69.789 \\
87.338 \\
68.632 \\
84.559 \\
48.532 \\
59.842 \\
61.451 \\
56.954 \\
70.473 \\
84.555\end{array}$ & $\begin{array}{r}84,1 \\
95,5 \\
112,1 \\
118,6 \\
92,7 \\
114,4 \\
66,3 \\
84,0 \\
83,8 \\
81,7 \\
93,0 \\
125,5\end{array}$ & $\begin{array}{r}77,9 \\
105,5 \\
97,3 \\
122,3 \\
95,7 \\
117,9 \\
67,6 \\
83,4 \\
85,7 \\
79,4 \\
98,2 \\
117,9\end{array}$ \\
\hline
\end{tabular}

Se hace patente, una vez más, la falta de homogeneidad de los valores representativos de actividades y estructuras entre las distintas Regiones. Del examen de las cifras referentes al consumo por hogar deducimos, en primer término, que el rango o amplitud del campo entre el consumo más alto -Vascongadas-Navarra- y el más bajo - Extremadura - es de 59. El consumo de la última supone el 53 por 100 del de las Vascongadas.

De las doce Regiones consideradas en la monografía, cuatro registran consumos superiores al nacional: Vascongadas-Navarra, Castilla la Nueva, Cataluña, Baleares y Canarias, por este orden. En la primera, el consumo es más elevado que el nacional en un 25 por 100, y en las otras tres, alrededor de una sexta parte en más.

Entre las ocho Regiones restantes, se pueden distinguir tres grupos:

a) El primero, formado por Aragón, Castilla la Vieja y Valen. cia, con un consumo cercano al nacional, aunque inferior a él entre el 5 y el 8 por 100; pero que, en relación con Vascongadas-Navarra y con las otras tres Regiones de mayor consumo, se sitúa una tercera y una quinta parte por debajo respectivamente.

b) El segundo, constituido por Andalucía, Galicia, León-Asturias y Murcia, de consumo más bajo, inferior al nacional en más de una sexta parte y en un 30 a 40 por 100 con respecto a las de mayor consumo.

c) El tercero lo forma Extremadura, con la tasa más baja de consumo. 
Si atendemos a los gastos de consumo por persona, las diferencias entre Regiones son menos marcadas. En este caso, el rango baja a 55. También son cuatro las Regiones que rebasan la cota nacional: Castilla la Nueva, en una cuarta parte; Vascongadas-Navarra y Cataluña-Baleares, en algo más de la sexta, y Aragón, en un 5 por 100.

Muy próximos al nacional se hallan los consumos de Valencia, Canarias y Castilla la Vieja. Puede distinguirse también otro grupo, formado por Galicia y León-Asturias, con un consumo inferior en una sexta parte al nacional, y un último grupo con Murcia, Andalucía y Extremadura; el consumo de las dos primeras es inferior al nacional en más de una cuarta parte y en una tercera parte el de la última.

En líneas generales, atendiendo a ambos consumos, pueden configurarse como zona de consumo máximo: Vascongadas-Navarra, Cataluña-Baleares y Castilla la Nueva, aparte las islas Canarias. En las dos primeras se concentra principalmente la actividad industrial del país, y en la segunda de ellas existen dos de las zonas turísticas de mayor importancia. El nivel de Castilla la Nueva se debe sobre todo al peso de Madrid, como gran urbe, capital de la Nación y cabecera de una comarca de reciente industrialización. Canarias destaca en el sector de servicios, en función de su desarrollo turístico, sin desdeñar su agricultura de exportación y su actividad industrial en determinados sectores.

Las tres primeras Regiones enumeradas en el párrafo anterior constituyen los vértices de un triángulo en el centro-nordeste de la península, que se completa y extiende a ambos lados con Aragón, Valencia y Castilla la Vieja, cuyos consumos son, como hemos visto, muy similares al nacional. De esta forma se configura un área territorial muy amplia con los consumos más altos de España.

Los consumos más bajos son los del noroeste, con Galicia y León-Asturias, y los del sur, con Murcia, Andalucía y Extremadura.

\section{En las Provincias}

El detalle provincial del consumo y los números índices correspondientes son los siguientes: 
REVISTA DB ESTUDIOS DE LA VIDA LOCAL

\begin{tabular}{|c|c|c|c|c|}
\hline & \multicolumn{2}{|c|}{$\begin{array}{l}\text { CIFRAS ABSOLUTAS } \\
\text { (PESETAS) }\end{array}$} & \multicolumn{2}{|c|}{$\begin{array}{l}\text { NUMEROS INDICES. BASE: } \\
\text { CONSUMO NACIONAL = } 100\end{array}$} \\
\hline & Por hogar & Por persona & Por hogar & Por persona \\
\hline $\begin{array}{cccccccc}\text { Alava } & \ldots & \ldots & \ldots & \ldots & \ldots & \ldots & \ldots\end{array}$ & 352.833 & 93.186 & 132,0 & 129,9 \\
\hline $\begin{array}{llllllll}\text { Albacete } & \ldots & \ldots & \ldots & \ldots & \ldots & \ldots & \ldots \\
\end{array}$ & 221.269 & 55.942 & 82,8 & 78,0 \\
\hline 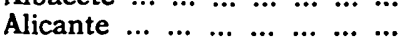 & 256.182 & 71.114 & $\begin{array}{l}95,0 \\
95,9\end{array}$ & 99,2 \\
\hline $\begin{array}{llllllll}\text { Almeria } & \ldots & \ldots & \ldots & \ldots & \ldots & \ldots & \ldots\end{array}$ & 215.155 & 54.370 & 80,5 & 75,8 \\
\hline $\begin{array}{lllllllll}\text { Avila } & \ldots & \ldots & \ldots & \ldots & \ldots & \ldots & \ldots & \ldots\end{array}$ & 195.960 & 56.789 & 73,3 & 79,2 \\
\hline $\begin{array}{cccccccc}\text { Badajoz } & \ldots & \ldots & \ldots & \ldots & \ldots & \ldots & \ldots\end{array}$ & 190.023 & 51.135 & 71,1 & 71,3 \\
\hline $\begin{array}{cccccccc}\text { Baleares } & \ldots & \ldots & \ldots & \ldots & \ldots & \ldots & \ldots\end{array}$ & 246.564 & 75.239 & 92,3 & 104,9 \\
\hline $\begin{array}{lllllll}\text { Barcelona } & \ldots & \ldots & \ldots & \ldots & \ldots & \ldots\end{array}$ & 325.685 & 89.017 & 121,9 & 124,1 \\
\hline $\begin{array}{llllllll}\text { Burgos } & \ldots & \ldots & \ldots & \ldots & \ldots & \ldots & \ldots\end{array}$ & 220.491 & 61.870 & 82,5 & 86,3 \\
\hline $\begin{array}{lllllllll}\text { Cáceres } & \ldots & \ldots & \ldots & \ldots & \ldots & \ldots & \ldots\end{array}$ & 158.917 & 44.619 & 59,5 & 62,5 \\
\hline $\begin{array}{llllllll}C a d i z & \ldots & \ldots & \ldots & \ldots & \ldots & \ldots & \ldots\end{array}$ & 243.245 & 57.938 & 91,0 & 80,8 \\
\hline $\begin{array}{lllllll}\text { Castellón } & \ldots & \ldots & \ldots & \ldots & \ldots & \ldots\end{array}$ & 205.010 & 64.439 & 76,7 & 89,9 \\
\hline 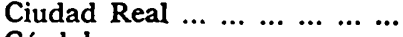 & 202.959 & 59.934 & 75,9 & 83,6 \\
\hline $\begin{array}{llllllll}\text { Córdoba } & \ldots & \ldots & \ldots & \ldots & \ldots & \ldots & \ldots \\
\text { Coruña }(\ldots \mathrm{La}) & \ldots & \ldots & \ldots & \ldots & \ldots & \ldots\end{array}$ & $\begin{array}{l}226.154 \\
257.174\end{array}$ & $\begin{array}{l}57.225 \\
65.407\end{array}$ & $\begin{array}{l}84,6 \\
96,2\end{array}$ & $\begin{array}{l}79,8 \\
912\end{array}$ \\
\hline $\begin{array}{llllllll} & & & & & \ldots & \ldots & \ldots\end{array}$ & 197.823 & 54.481 & 74,0 & 76,0 \\
\hline $\begin{array}{llllllll}\text { Gerona } & \ldots & \ldots & \ldots & \ldots & \ldots & \ldots & \ldots\end{array}$ & 288.114 & 76.425 & 107,8 & 106,6 \\
\hline $\begin{array}{cccccccc}\text { Granada } & \ldots & \ldots & \ldots & \ldots & \ldots & \ldots & \ldots\end{array}$ & 178.088 & 44.931 & 66,6 & 62,7 \\
\hline $\begin{array}{cccccc}\text { Guadalajara } & \ldots & \ldots & \ldots & \ldots & \ldots\end{array}$ & 206.863 & 60.128 & 77,4 & 83,8 \\
\hline $\begin{array}{ccccccc}\text { Guipúzcoa } & \ldots & \ldots & \ldots & \ldots & \ldots & \ldots\end{array}$ & 369.343 & 86.026 & 138,2 & 120,0 \\
\hline $\begin{array}{cccccccc}\text { Huelva } & \ldots & \ldots & \ldots & \ldots & \ldots & \ldots & \ldots\end{array}$ & 228.426 & 58.622 & 85,5 & 81,7 \\
\hline $\begin{array}{llllllll}\text { Huesca } & \ldots & \ldots & \ldots & \ldots & \ldots & \ldots & \ldots\end{array}$ & 252.302 & 69.217 & 94,9 & 96,5 \\
\hline $\begin{array}{lllllllll}\text { Jaén } & \ldots & \ldots & \ldots & \ldots & \ldots & \ldots & \ldots & \ldots\end{array}$ & 188.524 & 48.745 & 70,5 & 68,0 \\
\hline $\begin{array}{lllllllll}\text { León } & \ldots & \ldots & \ldots & \ldots & \ldots & \ldots & \ldots & \ldots\end{array}$ & 227.024 & 62.561 & 84,9 & 87,2 \\
\hline $\begin{array}{lllllllll}\text { Lérida } & \ldots & \ldots & \ldots & \ldots & \ldots & \ldots & \ldots & \ldots\end{array}$ & 243.817 & 69.237 & 912 & 96,7 \\
\hline $\begin{array}{llllllll}\text { Logroño } & \ldots & \ldots & \ldots & \ldots & \ldots & \ldots & \ldots\end{array}$ & 267.979 & 75.659 & 100,3 & 105,5 \\
\hline $\begin{array}{lllllllll}\text { Lugo } & \ldots & \ldots & \ldots & \ldots & \ldots & \ldots & \ldots & \ldots\end{array}$ & 160.529 & 43.422 & 60,1 & 60,5 \\
\hline $\begin{array}{llllllll}\text { Madrid } & \ldots & \ldots & \ldots & \ldots & \ldots & \ldots & \ldots\end{array}$ & 354.013 & 97.013 & 132,5 & 135,3 \\
\hline Málaga $\ldots \ldots \ldots \ldots \ldots$ & 234.648 & 57.410 & 87,8 & 80,1 \\
\hline $\begin{array}{llllllllll} & \text { Murcia } & \ldots & \ldots & \ldots & \ldots & \ldots & \ldots & \ldots & \ldots\end{array}$ & 217.406 & 57.343 & 81,3 & 80,0 \\
\hline $\begin{array}{llllllll}\text { Navarra } & \ldots & \ldots & \ldots & \ldots & \ldots & \ldots & \ldots\end{array}$ & 301.541 & 78.205 & 112,8 & 109,1 \\
\hline $\begin{array}{llllllll}\text { Orense } & \ldots & \ldots & \ldots & \ldots & \ldots & \ldots & \ldots\end{array}$ & 192.302 & 62.540 & 72,0 & 87,2 \\
\hline $\begin{array}{llllllll}\text { Oviedo } & \ldots & \ldots & \ldots & \ldots & \ldots & \ldots & \ldots\end{array}$ & 255.636 & 68.022 & 95,6 & 94,9 \\
\hline $\begin{array}{llllllll}\text { Palencia } & \ldots & \ldots & \ldots & \ldots & \ldots & \ldots & \ldots\end{array}$ & 220.272 & 55.740 & 82,4 & 77,7 \\
\hline $\begin{array}{llllll}\text { Palmas } & (\text { Las) } & \ldots & \ldots & \ldots & \ldots\end{array}$ & 317.954 & 70.202 & 119,0 & 97,9 \\
\hline $\begin{array}{lllllllllll} & \text { Pontevedra } & \ldots & \ldots & \ldots & \ldots & \ldots & \ldots & \ldots\end{array}$ & 237.611 & 59.611 & 88,9 & 83,1 \\
\hline 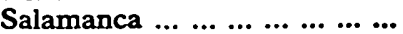 & 170.142 & 48.525 & 63,7 & 67,7 \\
\hline Santa Cruz de Tenerife ... & 282.563 & 69.361 & 105,7 & 96,7 \\
\hline $\begin{array}{lllllll}\text { Santander } & \ldots & \ldots & \ldots & \ldots & \ldots & \ldots\end{array}$ & 303.034 & 83.467 & 113,4 & 116,4 \\
\hline $\begin{array}{llllllll}\text { Segovia } & \ldots & \ldots & \ldots & \ldots & \ldots & \ldots & \ldots\end{array}$ & 215.620 & 61.395 & 80,7 & 85,6 \\
\hline $\begin{array}{lllllllll}\text { Sevilla } & \ldots & \ldots & \ldots & \ldots & \ldots & \ldots & \ldots & \ldots\end{array}$ & 251.776 & 61.837 & 94,2 & 86,2 \\
\hline $\begin{array}{lllllllll}\text { Soria } & \ldots & \ldots & \ldots & \ldots & \ldots & \ldots & \ldots & \ldots\end{array}$ & 234.067 & 68.002 & 87,6 & 94,8 \\
\hline $\begin{array}{ccccccc}\text { Tarragona } & \ldots & \ldots & \ldots & \ldots & \ldots & \ldots\end{array}$ & 268.978 & 73.866 & 100,6 & 103,0 \\
\hline 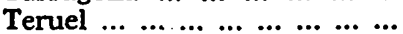 & 153.639 & 48.463 & 57,5 & 67,6 \\
\hline $\begin{array}{cccccccc}\text { Toledo } & \ldots & \ldots & \ldots & \ldots & \ldots & \ldots & \ldots\end{array}$ & 204.144 & 56.912 & 76,4 & 79,4 \\
\hline $\begin{array}{cccccccc}\text { Valencia } & \ldots & \ldots & \ldots & \ldots & \ldots & \ldots & \ldots\end{array}$ & 254.801 & 71.425 & 95,3 & 99,6 \\
\hline $\begin{array}{lllllll}\text { Valladolid } & \ldots & \ldots & \ldots & \ldots & \ldots & \ldots\end{array}$ & 249.645 & 67.657 & 93,4 & 94,3 \\
\hline 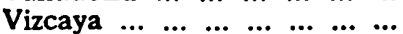 & 327.574 & 84.583 & 122,6 & 117,9 \\
\hline $\begin{array}{cccccccc}\text { Zamora } & \ldots & \ldots & \ldots & \ldots & \ldots & \ldots & \ldots\end{array}$ & 171.293 & 49.719 & 64,1 & 69.3 \\
\hline $\begin{array}{cccccccc}\text { Zaragoza } & \ldots & \ldots & \ldots & \ldots & \ldots & \ldots & \ldots\end{array}$ & 277.416 & 82.843 & 103,8 & 115,5 \\
\hline
\end{tabular}

En líneas generales, el análisis de la distribución provincial del consumo por hogar corrobora las conclusiones fijadas en el co- 
mentario sobre la distribución regional. El rango, en este caso, se ha ampliado a 81, diferencia entre el valor máximo - Guipúzcoay el mínimo - Teruel- El consumo de la primera es superior al de la última en un 142 por 100.

Tan sólo 13 Provincias tienen consumo superior al nacional. En su mayor parte se sitúan en los tres vértices del triángulo centronordeste, al que se ha hecho alusión con anterioridad. El primer vértice lo forman Guipúzcoa, Alava, Vizcaya y Navarra, enumeradas por orden de importancia; pero a ellas se unen Santander, Logroño y Zaragoza, que forman parte de Regiones de segundo nivel de consumo. Las tres Provincias Vascongadas registran el consumo más alto del país, superior en una tercera parte al nacional por lo que respecta a las dos primeras; Vizcaya lo supera en una quinta parte; Santander y Navarra, en un 10 por 100, y Zaraggza y Logroño lo rebasan muy ligeramente.

El segundo vértice lo constituye Madrid, con un consumo similar al de Alava. El tercero lo forman Barcelona, Gerona y Tarragona; la primera tiene un consumo semejante al de Vizcaya, mientras que el de las otras dos es más reducido, superando modestamente la cota nacional. Las Palmas y Santa Cruz de Tenerife, con consumo superior al nacional en el 19 y el 15 por 100 respectivamente, confirman el peso de las islas Canarias como zona propia.

Con consumo cercano al nacional, aunque inferior a él entre el 4 y el 9 por 100, hay 10 Provincias: La Coruña, Oviedo y Valladolid, que representan la expansión hacia el oeste de la zona del primer vértice; Lérida y Huesca, nexo de unión entre los vértices primero y tercero; Valencia, Alicante y Baleares, expansión hacia el sur de la zona del tercer vértice, y al sur, Cádiz y Sevilla, pertenecientes a una Región de bajo consumo, que se incorporan a un grupo superior.

Descrita esta amplia zona del país con consumos superiores o próximos al nacional, quedan 27 Provincias de bajo consumo que permiten, sin embargo, cierta graduación entre ellas, pudiéndose formar los siguientes grupos:

a) El primero, constituido por 12 Provincias, de consumo inferior al nacional entre una sexta y una quinta parte. Son: Pontevedra al oeste; Burgos, León, Palencia, Segovia y Soria en el valle del Duero; Albacete y Murcia al sureste, y Almería, Córdoba, Málaga y Huelva al sur.

b) El segundo, de nueve Provincias, con consumos inferiores 
en una cuarta parte o más al nacional. Son: Orense al oeste; Avila en el Duero; Badajoz, Ciudad Real, Cuenca, Guadalajara y Toledo en la submeseta sur; Castellón en el litoral mediterráneo, y Jaén al sur.

c) El tercero, con los más bajos consumos, inferiores al nacional en una tercera parte o más, lo constituyen: Lugo, Zamora, Salamanca y Cáceres al noroeste; Teruel, con la mínima, en Aragón, y Granada al sur.

Con referencia al consumo por persona, el rango es de 75, más amplio que el existente entre las Regiones, y expresa la diferencia entre el valor máximo - Madrid-y el mínimo - Lugo-.

El consumo superior al nacional se distribuye en forma más similar a la expuesta respecto al consumo por hogar. Son 12 las Provincias afectadas, con la sola diferencia de Baleares, que se incorpora al vértice catalán, y las Provincias Canarias, que pasan al grupo inferior. El consumo máximo corresponde a la Provincia de Madrid.

También se registra la existencia de 10 Provincias con consumo inferior al nacional, aunque próximo a él, que son casi las mismas enumeradas al analizar el consumo por hogar, con la sola diferencia de que las Canarias sustituyen a las Provincias andaluzas y Soria a las Baleares.

Cabe distinguir también los tres grupos de Provincias de bajo consumo, que son 28 en total, apreciándose ciertas diferencias en relación con los configurados al hablar del consumo por hogar. En el primero de ellos, formado por 13 Provincias, figuran: Orense y Pontevedra al noroeste; Burgos, León y Segovia en el Duero; Ciudad Real y Guadalajara en la submeseta sur; Castellón en el litoral mediterráneo; Murcia al sudeste, y Huelva, Cádiz, Sevilla y Málaga, en Andalucía, al sur. El segundo grupo, de tan sólo ocho Provincias, está constituido por: Avila y Palencia en el Duero; Badajoz, Albacete, Cuenca y Toledo en la submeseta sur, y Almería y Córdoba en el sur. El tercer grupo, de consumos mínimos, tiene una composición similar a la descrita en relación con el consumo por hogar, con la sola adición de la Provincia de Jaén.

Quedan de esta forma suficientemente definidas las zonas de máximos y mínimos consumos en la geografía nacional. 
3. EN Los Municipios, SEGÚN SUS NIVELES de POBLACIÓN

El gasto anual medio de consumo, por grupos de Municipios, presenta estos valores:

\begin{tabular}{|c|c|c|c|c|}
\hline \multirow{4}{*}{$\begin{array}{ccccc}\text { Hasta } 2.000 \text { habitantes } & \ldots & \ldots \\
\text { De } 2.001 \text { a } 10.000 & \ldots & \ldots & \ldots & \ldots \\
\text { De } 10.001 \text { a } 50.000 & \ldots & \ldots & \ldots & \ldots \\
\text { Más de } 50.000 \text { habitantes. } & \text { Ca- } \\
\text { pitales de Provincia } & \ldots & \ldots\end{array}$} & \multicolumn{2}{|c|}{$\begin{array}{l}\text { CIFRAS ABSOLUTAS } \\
\text { (PESETAS) }\end{array}$} & \multicolumn{2}{|c|}{$\begin{array}{l}\text { NUMEROS INDICES. BASE: } \\
\text { CONSUMO NACIONAL }=100\end{array}$} \\
\hline & Por hogar & Por persona & Por hogar & Por persona \\
\hline & $\begin{array}{l}190.003 \\
250.986 \\
252.826\end{array}$ & $\begin{array}{l}54.169 \\
56.164 \\
64.665\end{array}$ & $\begin{array}{l}71,1 \\
77,1 \\
94,6\end{array}$ & $\begin{array}{l}75,5 \\
78,3 \\
90,2\end{array}$ \\
\hline & 321.822 & 86.629 & 120,4 & 120,8 \\
\hline
\end{tabular}

Los consumos más elevados corresponden a los Municipios de mayor importancia demográfica. Las capitales de Provincia y los Municipios mayores de 50.000 habitantes ofrecen un consumo superior al nacional en una quinta parte. Llama la atención el valor similar entre el consumo por hogar y por persona.

En los Municipios de población comprendida entre 10.000 y 50.000 habitantes, el consumo, aunque inferior, se aproxima a la cota nacional, y en ellos el consumo por persona aparece por debajo del consumo por hogar.

Los restantes grupos de Municipios tienen consumos inferiores al nacional en una cuarta parte, aproximadamente, y el consumo por persona es relativamente superior al del hogar.

Se da, pues, una relación directa entre el volumen de población y la cuantía del consumo.

El detalle del consumo por hogar y por persona en las distintas Regiones, atendiendo a los grupos de Municipios considerados, es éste:

\begin{tabular}{|c|c|c|c|c|}
\hline & $\begin{array}{c}\text { Hasta } \\
2.000\end{array}$ & $\begin{array}{l}\text { De } 2.001 \\
\text { a } 10.000\end{array}$ & $\begin{array}{l}\text { De } 10.001 \\
\text { a } 50.000\end{array}$ & $\begin{array}{l}\text { Más de } \\
50.000 \text { y } \\
\text { capitales } \\
\text { Provincia }\end{array}$ \\
\hline & \multicolumn{4}{|c|}{$\begin{array}{c}\text { Gasto anual medio de consumo por hogar. } \\
\text { Cifras absolutas (pesetas) }\end{array}$} \\
\hline 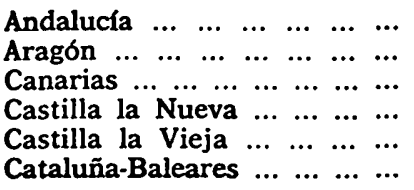 & $\begin{array}{l}174.777 \\
168.881 \\
- \\
182.505 \\
185.782 \\
271.112\end{array}$ & $\begin{array}{l}173.429 \\
241.653 \\
226.306 \\
211.035 \\
253.174 \\
249.558\end{array}$ & $\begin{array}{l}212.036 \\
220.984 \\
270.164 \\
240.839 \\
277.658 \\
309.639\end{array}$ & $\begin{array}{l}282.880 \\
329.667 \\
354.319 \\
362.190 \\
307.373 \\
324.554\end{array}$ \\
\hline
\end{tabular}




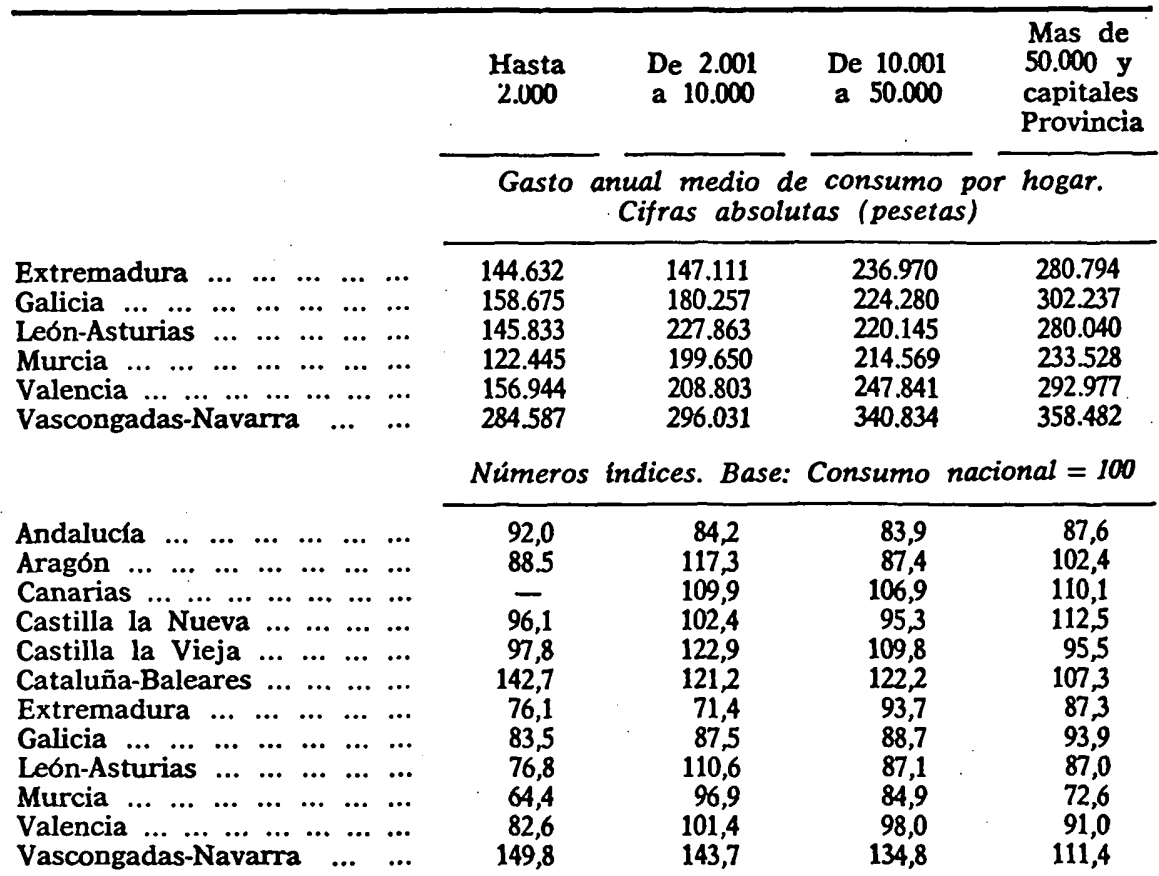

Gasto anual medio de consumo por persona. Cifras absolutas (pesetas)

$\begin{array}{llllllll}\text { Andalucia } & \ldots & \ldots & \ldots & \ldots & \ldots & \ldots\end{array}$

Aragón $\quad \ldots \begin{array}{llllllllll} & \ldots & \ldots & \ldots & \ldots & \ldots & \ldots & \ldots\end{array}$

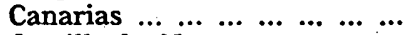

Castilla la Nueva $\ldots . . . . . . . . .$.

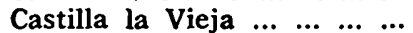

Cataluña-Baleares $\ldots . . . . . . . . .$.

$\begin{array}{llllll} & \text { Extremadura } & \ldots & \ldots & \ldots & \ldots\end{array}$

$\begin{array}{lllllllll}\text { Galicia } & \ldots & \ldots & \ldots & \ldots & \ldots & \ldots & \ldots & \ldots\end{array}$

$\begin{array}{lllllll}\text { León-Asturias } & \ldots & \ldots & \ldots & \ldots & \ldots\end{array}$

$\begin{array}{lllllllll}\text { Murcia } & \ldots & \ldots & \ldots & \ldots & \ldots & \ldots & \ldots\end{array}$

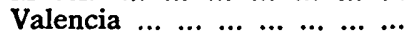

Vascongadas-Navarra $\quad . . . \quad \ldots$

$\begin{array}{lllllllll}\text { Andalucía } & \ldots & \ldots & \ldots & \ldots & \ldots & \ldots\end{array}$

$\begin{array}{lllllllll}\text { Aragón } & \ldots & \ldots & \ldots & \ldots & \ldots & \ldots & \ldots & \ldots\end{array}$

$\begin{array}{lllllllll}\text { Canarias } & . . & \ldots & \ldots & \ldots & \ldots & \ldots & \ldots & \ldots\end{array}$

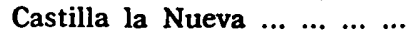

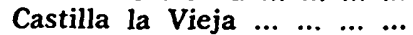

$\begin{array}{llllll}\text { Cataluña-Baleares } & . . & \ldots & \ldots & \ldots & \ldots\end{array}$

$\begin{array}{lllllll}\text { Extremadura } & \ldots & \ldots & \ldots & \ldots & \ldots\end{array}$

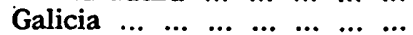

$\begin{array}{lllllll}\text { León-Asturias } & \ldots & \ldots & \ldots & \ldots & \ldots\end{array}$

$\begin{array}{lllllllll}\text { Murcia } & \ldots & \ldots & \ldots & \ldots & \ldots & \ldots & \ldots & \ldots\end{array}$

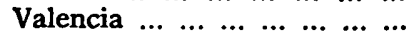

$\begin{array}{lllll}\text { Vascongadas-Navarra } & \ldots & \ldots\end{array}$

$\begin{array}{lrr}44.551 & 50.735 & 70.267 \\ 66.421 & 62.038 & 91.643 \\ 54.720 & 62.255 & 81.923 \\ 56.627 & 66.983 & 100.057 \\ 70.116 & 73.616 & 80.863 \\ 71.604 & 80.086 & 91.929 \\ 42.477 & 59.649 & 67.584 \\ 49.927 & 57.845 & 78.420 \\ 60.746 & 57.515 & 76.814 \\ 52.399 & 57.162 & 60.244 \\ 64.426 & 67.075 & 82.274 \\ 74.947 & 82.853 & 92.736\end{array}$

Números indices. Base: Consumo nacional $=100$

\begin{tabular}{rrrr}
\hline 84,5 & 79,3 & 78,5 & 81,1 \\
105,8 & 118,3 & 99,0 & 105,0 \\
- & 97,4 & 96,3 & 94,6 \\
98.4 & 100,8 & 103,6 & 115,5 \\
100,7 & 124,8 & 120,0 & 93,3 \\
128,4 & 127,5 & 123,8 & 106,1 \\
74,9 & 75,6 & 92,2 & 78,0 \\
68,9 & 88,9 & 89,5 & 90,5 \\
78,3 & 108,2 & 88,9 & 88,7 \\
52,6 & 93,3 & 88,4 & 69,5 \\
90,1 & 114,7 & 103,7 & 95,0 \\
133,2 & 133,4 & 128,1 & 107,1 \\
\hline
\end{tabular}


Al examinar los índices obtenidos para el consumo por hogar se advierte, en primer término, que en los Municipios pequeños los consumos superiores al nacional destacan sobremanera en las dos Regiones de máximo grado de industrialización: Vascongadas-Navarra y Cataluña-Baleares, con tasas superiores a las de las propias Regiones incluso. Se aproximan a la cota nacional los consumos de estos Municipios en ambas Castillas y en Andalucía. En las restantes Regiones, el consumo puede estimarse como bajo, dando Murcia el mínimo.

En los Municipios del grupo inmediato se generalizan los consumos superiores al nacional, pues comprenden ocho Regiones, que se extienden sobre gran parte del territorio nacional, y aún Murcia se aproxima a la cota nacional. Tan sólo los Municipios de este grupo en Galicia, Extremadura y Andalucía dan consumos más bajos.

En los Municipios de población comprendida entre 10.000 y 50.000 habitantes, los consumos superiores al nacional se limitan a cuatro Regiones, aunque se acercan a la cota nacional tres más. En las cinco restantes, los consumos no son demasiado bajos. Estas mismas características, en términos generales, son válidas para los grandes Municipios.

La evolución del consumo de los grupos de Municipios de Cataluña-Baleares y Vascongadas-Navarra nos permite afirmar que a medida que aumenta el tamaño demográfico de los Municipios disminuye la cuantía del consumo por hogar. Se advierte, sin embargo, cierta uniformidad en el consumo de los Municipios de todos los niveles en Andalucía, Canarias y Galicia. En las demás Regiones predominan, en unos casos, los consumos de algún grupo de Municipios en concreto.

Aunque la distribución de estos grupos de Municipios por Provincias daría posiblemente tasas más bajas de consumo, parece deducirse del detalle regional un acercamiento paulatino de las tasas de consumo por Municipios a cuantías más uniformes; o, lo que es lo mismo, una tendencia a la homogeneidad en el consumo, aun contando con las distintas características de las Regiones.

Es evidente que en la determinación de la cuantía del consumo por persona, en los grupos de Municipios considerados, tiene notable influencia la composición del hogar, en cuanto al número de miembros que lo forman; de aquí se deducen ciertas discre- 
pancias, dentro de una tónica general uniforme, entre la evolución del consumo por hogar y por persona.

Es de subrayar el alto nivel de consumo por persona en los grandes Municipios de Castilla la Nueva, Cataluña-Baleares, Vascongadas-Navarra y Aragón, en las que se ubican las más importantes ciudades españolas.

\section{LA EVOLUCION DEL CONSUMO POR PERSONA EN LOS CONJUNTOS URBANO Y SUBURBANO}

Resulta de extraordinario interés conocer la evolución del consumo por persona, registrada a través de las distintas encuestas de presupuestos familiares realizadas, y especialmente su discriminación en los cinco grupos clásicos en que se presenta ordinariamente la composición del presupuesto familiar y los índices de precios al consumo.

A estos efectos se presentan los datos en tres conjuntos: el nacional, el suburbano y el urbano. El último de ellos está formado por las capitales de Provincia y los Municipios, no capitales, mayores de 50.000 habitantes. El detalle apuntado es el siguiente, expresado en pesetas.

\begin{tabular}{|c|c|c|c|c|}
\hline & & A & $\mathbf{0} \mathbf{s}$ & \\
\hline . & 1958 & 1964 & 1968 & $1973-74$ \\
\hline & & Conje & nacional & \\
\hline $\begin{array}{llllll}\text { Alimentación } & \ldots & \ldots & \ldots & \ldots & \ldots\end{array}$ & 5.963 & 9.729 & 14.391 & 27.274 \\
\hline Vestido y calzado ... ... ... ... & 1.464 & 2.975 & 4.386 & 5.524 \\
\hline $\begin{array}{lllllllll}\text { Vivienda } & \ldots & \ldots & \ldots & \ldots & \ldots & \ldots & \ldots\end{array}$ & 538 & 1.475 & 3.329 & 8.282 \\
\hline Gastos de casa $\ldots \ldots \ldots \ldots$ & 888 & 1.842 & 2.636 & 7.969 \\
\hline $\begin{array}{ccccccc}\text { Gastos } & \text { de } & \text { verano } & \text { у } & \text { vaca- } \\
\text { ciones } & \ldots & \ldots & \ldots & \ldots & \ldots & \ldots\end{array}$ & 1.926 & 3.953 & 7.673 & 22.661 \\
\hline Consumo total $\ldots \ldots$ & 10.765 & 19.974 & 32.415 & 71.715 \\
\hline & & Conjus & suburbano & \\
\hline $\begin{array}{llllll}\text { Alimentación } & \ldots & \ldots & \ldots & \ldots & \ldots\end{array}$ & 5.389 & 8.319 & 12.065 & 25.294 \\
\hline Vestido y calzado $\ldots \ldots \ldots \ldots$ & 1.243 & 2.343 & 3.627 & 4.597 \\
\hline $\begin{array}{llllllll}\text { Vivienda } & \ldots & \ldots & \ldots & \ldots & \ldots & \ldots & \ldots\end{array}$ & 409 & 860 & 2.202 & 6.137 \\
\hline $\begin{array}{l}\text { Gastos de casa } \ldots . . . \\
\text { Gastos de verano y }\end{array}$ & 743 & 1.221 & 1.765 & 6.008 \\
\hline 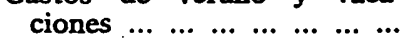 & 1.344 & 2.446 & 4.964 & 17.119 \\
\hline Consumo total ... ... ... & 9.128 & 15.189 & 24.623 & 59.155 \\
\hline
\end{tabular}




\begin{tabular}{|c|c|c|c|c|}
\hline \multirow[b]{4}{*}{ 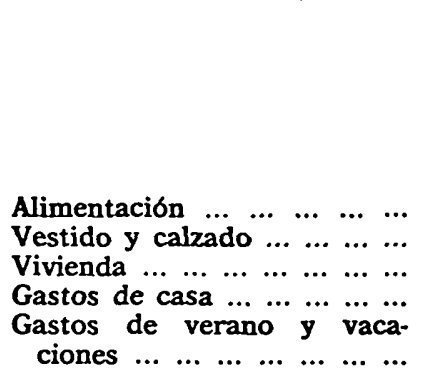 } & \multicolumn{4}{|c|}{$\begin{array}{llll}A & \mathbf{N} & \mathbf{O} & \mathbf{S}\end{array}$} \\
\hline & 1958 & 1964 & 1968 & $1973-74$ \\
\hline & \multicolumn{4}{|c|}{ Conjunto urbano } \\
\hline & $\begin{array}{r}6.096 \\
1.511 \\
556 \\
915 \\
\\
2.022\end{array}$ & $\begin{array}{r}10.755 \\
3.434 \\
1.923 \\
2.992 \\
\\
5.048\end{array}$ & $\begin{array}{r}15.900 \\
4.879 \\
4.067 \\
3.209 \\
9.450\end{array}$ & $\begin{array}{r}29.632 \\
6.618 \\
10.826 \\
10.296 \\
29.251\end{array}$ \\
\hline Consumo total $\ldots \ldots \ldots$ & 11.100 & 23.452 & 37.505 & 86.269 \\
\hline
\end{tabular}

Se observa el fuerte incremento, en pesetas nominales, del consumo por persona en la última encuesta, que es un indicador suficiente de los altos niveles alcanzados por el Producto Nacional Bruto, la Renta total y la Renta por habitante.

Podemos convertir, para'mejor conocer la apuntada evolución, la tabla transcrita, en pesetas nominales, en otra de pesetas constantes $y$, sobre ésta, hallar los índices del consumo anual medio por persona, tomando como base el año $1958=100$. La distribución es la siguiente:

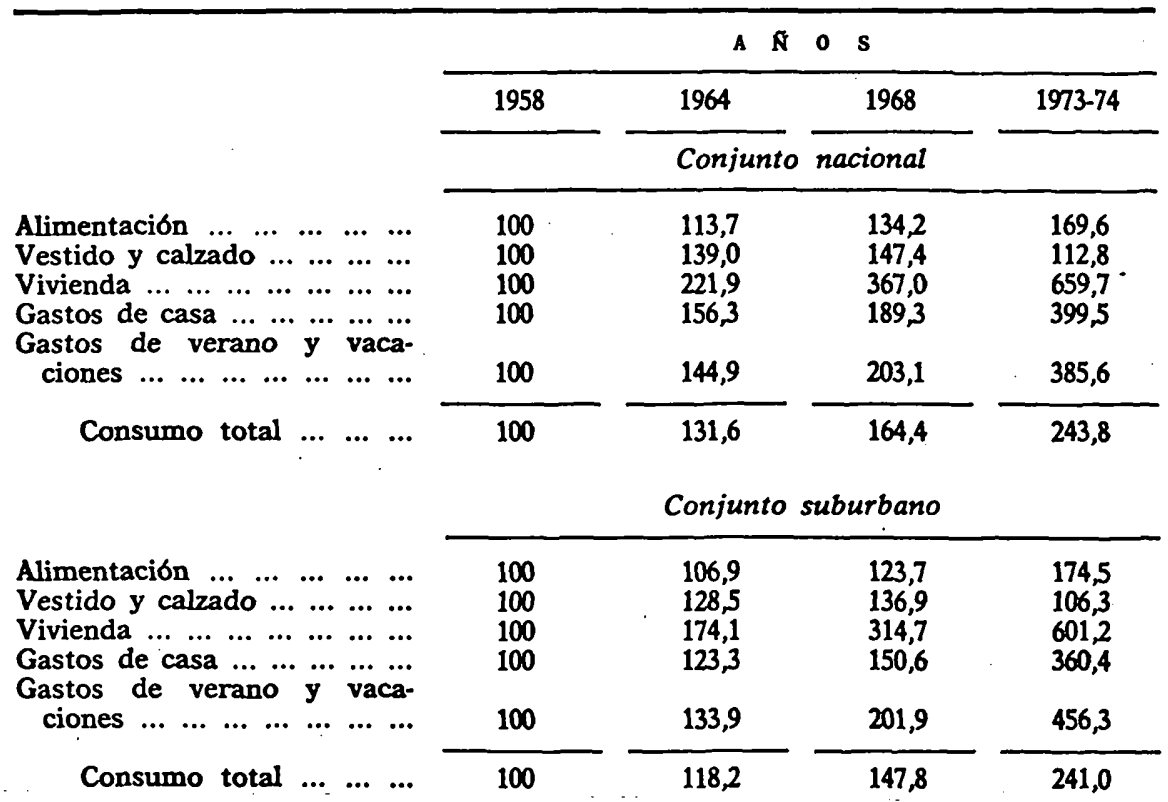




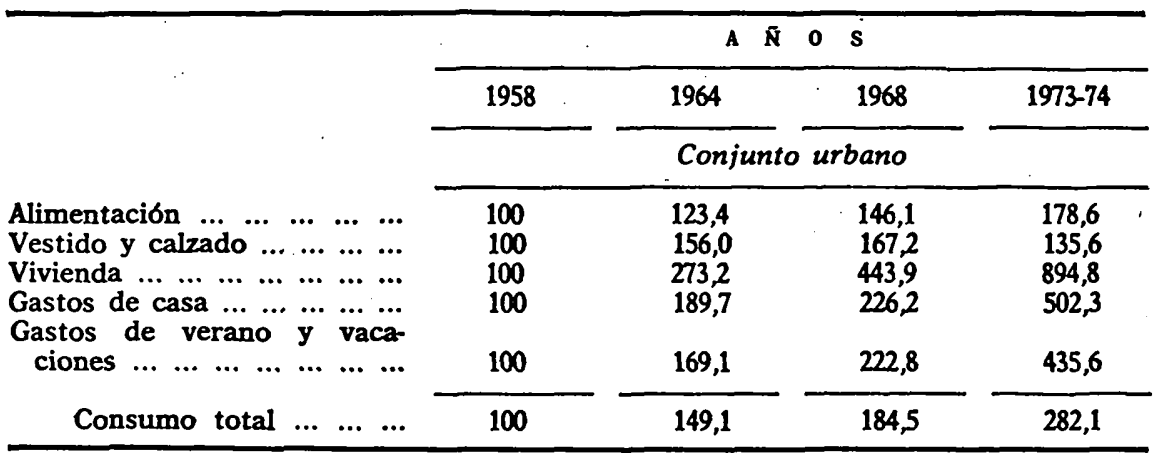

Se observa que el consumo total se ha multiplicado por 2,4 en el conjunto nacional y en el suburbano, y por 2,8 en el conjunto urbano. Se registra, de otra parte, unos fuertes incrementos del gasto en vivienda, gastos de casa y gastos de verano y vacaciones, que afectan tanto al conjunto urbano como al suburbano, siendo de subrayar el fuerte ritmo con que la población suburbana se ha incorporado a la «civilización del ocio». Por el contrario, se registra una disminución acusada en los gastos de vestido y calzado y un incremento moderado en los de alimentación.

Finalmente, es interesante conocer la evolución interna del consumo total porque es un indicador eficaz de la evolución del nivel de vida. En la siguiente tabla se presentan los porcentajes del consumo total para los años y conjuntos considerados:

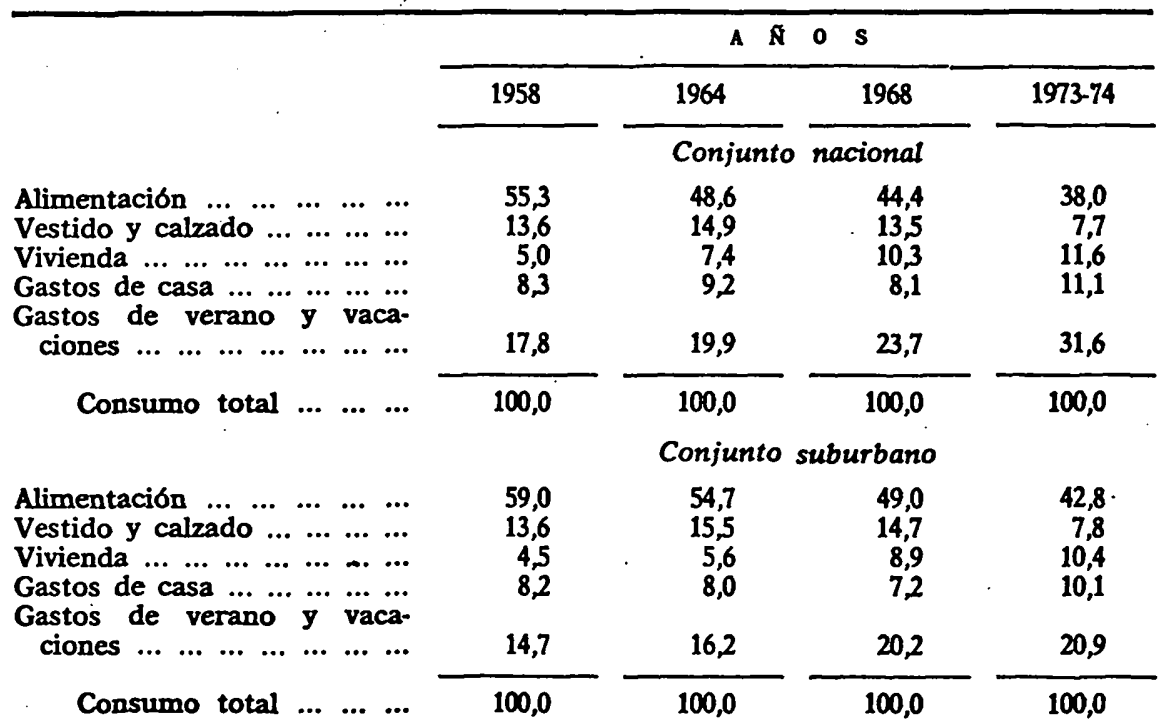




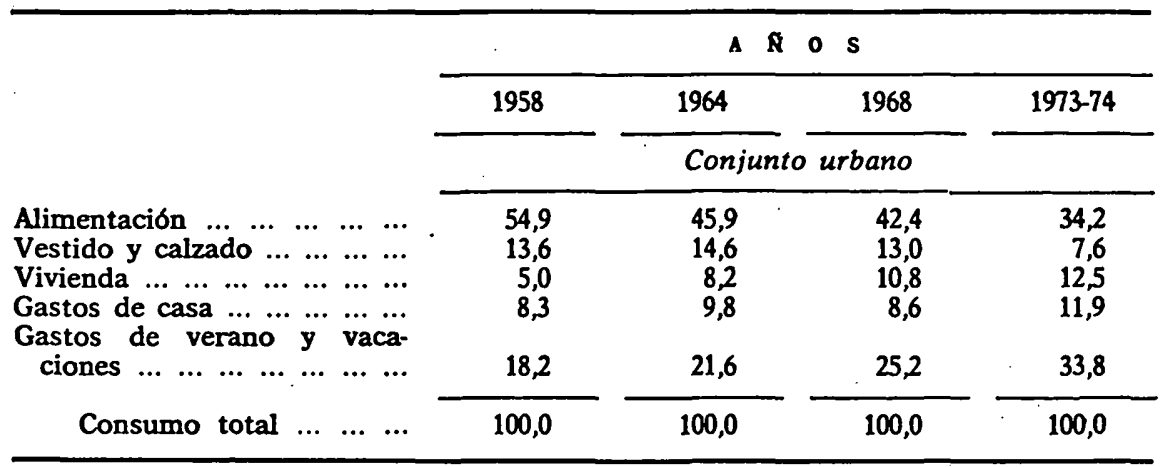

El peso de la alimentación en el consumo total ha disminuido paulatinamente desde 1958; pero de manera más acusada en el período 1968-1974. Este fenómeno afecta esencialmente al conjunto urbano; en el conjunto suburbano, aunque el ritmo descendente de la alimentación es claro, se mantiene aún a más alto nivel. De igual forma se ha puesto de manifiesto en el indicado período un acentuado descenso de los gastos de calzado y vestido, que en 1974 representan tan sólo la mitad del consumo habido en 1968.

Por el contrario, se han duplicado al menos los gastos en vivienda, se incrementan rápidamente los gastos de casa; y debe subrayarse el hecho del patente aumento de los gastos de verano y vacaciones, que constituyen un fenómeno generalizado que afecta a todos los estratos sociales.

En su conjunto, esta evolución que se opera en los componentes del consumo total constituye un indicador de la elevación del nivel de vida de la población española. 
REVL-1975, núm. 186. BALLESTER ROS, IGNACIO. EL CONSUMO EN LOS HOGARES ESPAÑOLES...

REVL-1975, núm. 186. BALLESTER ROS, IGNACIO. EL CONSUMO EN LOS HOGARES ESPAÑOLES... 
REVL-1975, núm. 186. BALLESTER ROS, IGNACIO. EL CONSUMO EN LOS HOGARES ESPAÑOLES...

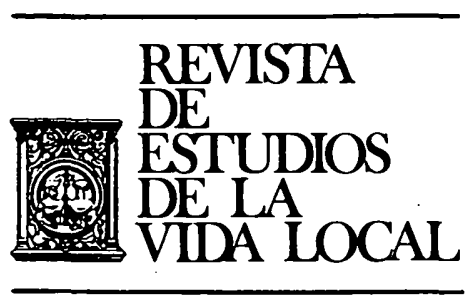

IV. JURISPRUDENCIA

REVL-1975, núm. 186. BALLESTER ROS, IGNACIO. EL CONSUMO EN LOS HOGARES ESPAÑOLES... 
REVL-1975, núm. 186. BALLESTER ROS, IGNACIO. EL CONSUMO EN LOS HOGARES ESPAÑOLES...

REVL-1975, núm. 186. BALLESTER ROS, IGNACIO. EL CONSÜMO EN LOS HOGARES ESPAÑOLES... 